\title{
High flux femtosecond x-ray emission from the electron-hose instability in laser wakefield accelerators
}

\author{
C. F. Dong, ${ }^{1,2,3,{ }^{*}}$ T. Z. Zhao, ${ }^{3}$ K. Behm, ${ }^{3}$ P. G. Cummings, ${ }^{3}$ J. Nees, ${ }^{3}$ A. Maksimchuk, ${ }^{3}$ \\ V. Yanovsky, ${ }^{3}$ K. Krushelnick, ${ }^{3}$ and A. G. R. Thomas ${ }^{3,4}$ \\ ${ }^{1}$ Princeton Plasma Physics Laboratory, Princeton University, Princeton, New Jersey 08540, USA \\ ${ }^{2}$ Department of Astrophysical Sciences, Princeton University, Princeton, New Jersey 08544, USA \\ ${ }^{3}$ Center for Ultrafast Optical Science, University of Michigan, Ann Arbor, Michigan 48109, USA \\ ${ }^{4}$ Physics Department, Lancaster University, Lancaster LA1 4YB, United Kingdom
}

\section{(Received 22 August 2016; published 24 April 2018)}

\begin{abstract}
Bright and ultrashort duration x-ray pulses can be produced by through betatron oscillations of electrons during laser wakefield acceleration (LWFA). Our experimental measurements using the HERCULES laser system demonstrate a dramatic increase in x-ray flux for interaction distances beyond the depletion/ dephasing lengths, where the initial electron bunch injected into the first wake bucket catches up with the laser pulse front and the laser pulse depletes. A transition from an LWFA regime to a beam-driven plasma wakefield acceleration regime consequently occurs. The drive electron bunch is susceptible to the electronhose instability and rapidly develops large amplitude oscillations in its tail, which leads to greatly enhanced $\mathrm{x}$-ray radiation emission. We measure the $\mathrm{x}$-ray flux as a function of acceleration length using a variable length gas cell. 3D particle-in-cell simulations using a Monte Carlo synchrotron x-ray emission algorithm elucidate the time-dependent variations in the radiation emission processes.
\end{abstract}

DOI: 10.1103/PhysRevAccelBeams.21.041303

Laser wakefield acceleration (LWFA) is a technique that has experimentally demonstrated electron acceleration to greater than $\mathrm{GeV}$ energies over $\mathrm{cm}$-scale acceleration lengths [1,2]. In LWFA, electrons are accelerated by strong electric fields associated with plasma waves generated by a high-power, short pulsed laser. These electrons can produce energetic $\mathrm{x}$-ray emission via oscillations of the electron beam in the transverse fields of the plasma wake [3] and may also be used as a diagnostic of the electron dynamics in the plasma wake [4-6]. Experimental measurements of such radiation generated by betatron oscillations in an LWFA have demonstrated bright and well-collimated beams of $x$ rays [7-10]. The $x$-ray generation mechanism has been studied theoretically in an ion channel [11-14] or bubble $[15,16]$. These $\mathrm{x}$-ray sources are particularly appealing for ultrafast imaging applications due to their femtosecond duration $[7,17]$.

Recent studies have shown that a transition from an LWFA to a beam-driven plasma wakefield accelerator (PWFA) occurs when the first group of electrons catches up with the laser front, following depletion of the laser

\footnotetext{
*dcfy@pppl.gov
}

Published by the American Physical Society under the terms of the Creative Commons Attribution 4.0 International license. Further distribution of this work must maintain attribution to the author(s) and the published article's title, journal citation, and DOI. pulse and dephasing [18]. The transverse ponderomotive force of the laser pulse no longer sustains the wakefield, which is now generated by the electron beam. Deviations of the electron beam through interaction with the weak laser field plants the seed for the electron-hose instability [19]. In general, this instability leads to spatiotemporally growing oscillations of the beam centroid along its axis of propagation and can limit the useful acceleration length, as well as increasing the beam emittance. Later studies that investigated this instability for beam-driven plasma accelerators showed that the growth rate was reduced when nonadiabatic ion-channel formation and magnetized plasma was taken into account $[20,21]$. For typical LWFA experimental conditions, however, the growth rate can be sufficiently rapid to produce violent electron oscillations in the laser polarization direction that eventually cause beam breakup and termination of the wakefield [22]. Although these instabilities are undesirable for some plasma based accelerator applications, they will increase the radiated flux dramatically due to the large-amplitude transverse oscillations of the electron beam resulting from this instability. A similar phenomenon is laser beam hosing [23], which has been shown in simulations to increase x-ray flux [24]. Another mechanism is resonant interaction of the electron beam with the laser fields, which has also been shown to enhance the x-ray production $[15,25]$. Although the $\mathrm{x}$ rays produced by the instability have a larger source size relative to those produced in the laser driven wakefield, they maintain 


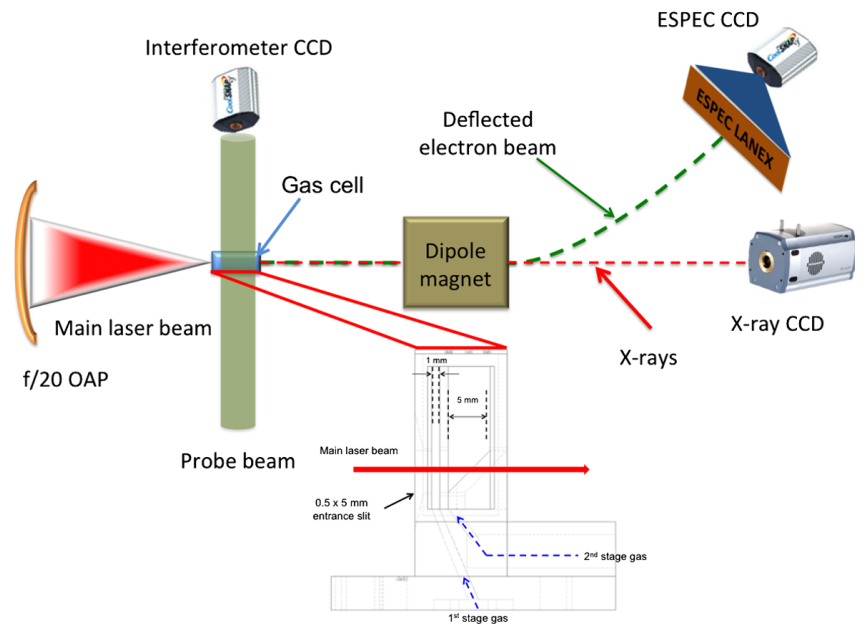

FIG. 1. Schematic of the experimental setup. The main beam is focused using an $f / 20$ off-axis paraboloid onto a two-stage gas cell. Electrons exiting the gas cell were swept by a dipole magnet and imaged using a LANEX screen in conjunction with a CCD camera. X rays were measured $2.5 \mathrm{~m}$ downstream with an X-ray camera. A two-stage, variable length gas cell is zoomed in. The acceleration length can be changed by adjusting the vertical height of the gas cell with respect to the main laser beam (as a result of the 45 degree incline in the second stage). The dashed arrows show the path of gas flow into the cell.

their femtosecond duration and have much larger flux and therefore may be useful for some ultrafast pumpprobe applications.

In this paper, we measure the x-ray flux as a function of acceleration length in an LWFA using a variable length gas cell with fixed density. We show that for propagation extending many times the dephasing length, where the electron beam is susceptible to the electron-hose instability $[19,21,22,26]$, the $x$-ray flux is consequently increased dramatically due to the violent wiggling of the high energy electron beam. The propagation length-dependent variations in betatron radiation are also demonstrated in detail by 3D OsIRIs particle-in-cell simulations with a Monte Carlo synchrotron $\mathrm{x}$-ray emission algorithm.

The experiment was performed using the $800 \mathrm{~nm}$ wavelength, Ti:sapphire HERCULES laser facility at the University of Michigan. Figure 1 shows the experimental setup. The horizontally polarized laser beam, delivering $1.6 \mathrm{~J}$ in a $34 \mathrm{fs}$ pulse (full width at half maximum, FWHM, in intensity), was focused using an $f / 20$ off-axis paraboloidal mirror to a vacuum beam waist $w_{0}=26 \mu \mathrm{m}$ (at $1 / e^{2}$ of peak intensity) with on-target peak intensities of $2 \times 10^{19} \mathrm{~W} \mathrm{~cm}^{-2}$, corresponding to a normalized field strength $a_{0}=3$. A 3D-printed variable-length two-stage gas cell was used as the target [27]. The cell was composed of a $1 \mathrm{~mm}$ injection stage, a $0.5 \mathrm{~mm}$ divider slit for stage separation, and an adjustable 0-5 mm acceleration stage, which can be adjusted by varying the height of the gas cell. The injection stage was filled with a mixed gas $\left(97.5 \% \mathrm{He}\right.$ and $\left.2.5 \% \mathrm{~N}_{2}\right)$ [28] and the acceleration

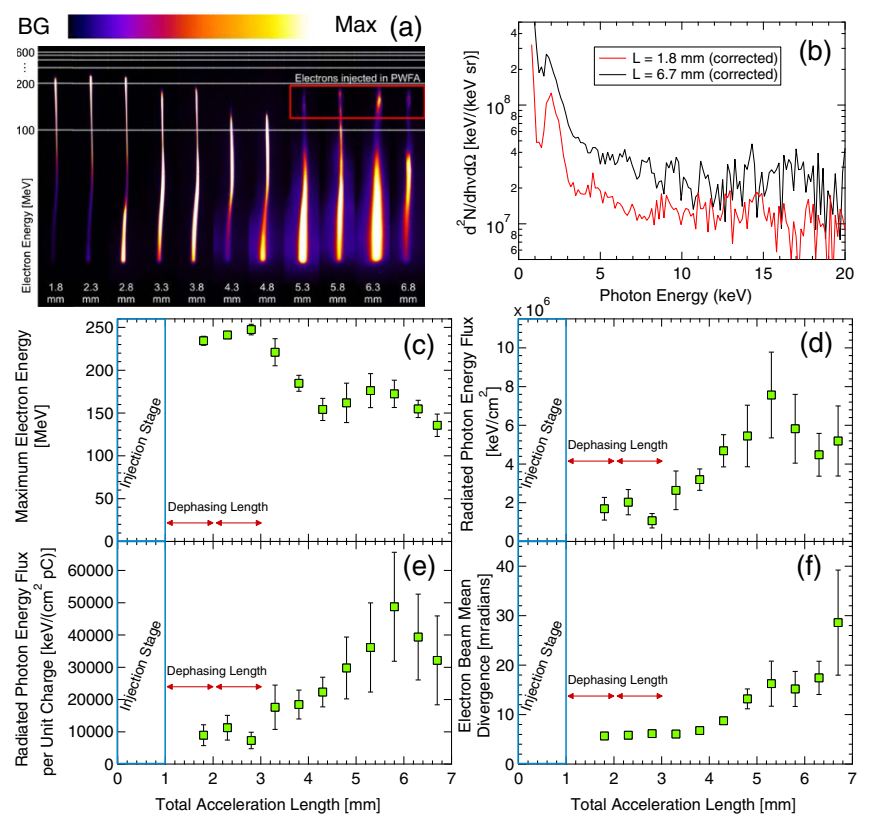

FIG. 2. (a) Typical electron $p_{\|}-p_{\perp}$ momentum distributions for various length gas cells measured using a magnetic spectrometer. (b) X-ray spectra at two selected acceleration lengths with quantum efficiency and filter corrections applied. (c) Maximum electron energy. (d) Total radiated photon energy (TRPE). (e) TRPE per unit charge. (f) Electron beam mean divergence. Parts (d) and (e) show a dramatic increase as a function of acceleration length at fixed density. Error bars denote the standard error of the mean.

stage with pure helium. In this experiment, the average peak electron density, determined interferometrically, across the entire gas cell was fixed at $(1.0 \pm 0.2) \times 10^{19} \mathrm{~cm}^{-3}$ for all lengths.

The energy of the electron beam was measured using a $0.8 \mathrm{~T}$ magnetic dipole which dispersed the beam onto a LANEX scintillator screen. X-ray flux was measured using an Andor iKon-M BR-DD camera, with X-ray sensitivity up to $30 \mathrm{keV}$ and a $13 \times 13 \mathrm{~mm}^{2}$ chip size with $1 \mathrm{M}$ pixels, which was placed $2.5 \mathrm{~m}$ downstream from the interaction region and shielded from the laser light with a $20 \mu \mathrm{m} \mathrm{Be}$ window. The single hit spectrum was measured using standard single photon counting techniques [29] and adjusted for the quantum efficiency (QE) of the camera and any filters the X-ray beam passes through [30], as in Fig. 2(b). The notch in the "corrected" spectra is not a real feature but rather an artifact of uncertainty in the corrections for filter absorption and QE [30].

Our main experimental result is a measurement of the photon energy flux as a function of the gas cell acceleration length. The average laser power was $46 \mathrm{TW}$, giving an estimated dephasing and depletion length of 1 and $1.9 \mathrm{~mm}$, respectively [31]. As shown in Fig. 2(a), quasimonoenergetic electron bunches are generated that subsequently dephase and broaden in energy spread. When the 
acceleration length exceeds two dephasing lengths, ${ }^{1}$ the laser energy is depleted for our conditions and the electron beam propagates past the laser front, meaning that it transitions

to a beam driven wakefield accelerator [18], as evidenced by a second population of electrons being accelerated. In the angular direction, the divergence of the electron bunch is observed to increase considerably after this point [Fig. 2(f)]. Figure 2(c) shows the electron peak energy from this data averaged over approximately ten shots at each length.

The photon energy flux was simultaneously measured by integrating the signal on the X-ray CCD camera. As can be seen in Fig. 2(d), greater than a factor of 3 increase in the photon energy flux occurs on average as the accelerator transitions to beam driven acceleration, albeit with a constant flux to within error at the longest lengths, suggesting a saturation of the effect. Figure 2(e) shows the $\mathrm{x}$-ray flux per unit charge, for electrons with energy $>100 \mathrm{MeV}$ only, indicating this trend is not simply due to an increase in trapped charge. Although the overall beam charge increases with length as well, the bulk of the electrons are decelerated beyond two dephasing lengths and so the high energy component, which contributes predominantly, decreases in charge. The x-ray spectrum at two selected acceleration lengths, Fig. 2(b), is also enhanced by more than a factor of 3 on average, consistent with those trends shown in Figs. 2(d) and 2(e).

This dramatic increase in x-ray flux can be explained by the onset of the electron beam hosing instability. This leads to large amplitude oscillations of the electron beam as indicated by the beam mean divergence based on the average integrated width of the electron beam as a function of propagation length [Fig. 2(f)]. Since the power radiated by a charge performing large amplitude betatron oscillations scales as $P \simeq r_{e} m_{e} c^{3} \gamma^{4} k_{\beta}^{4} r_{\beta}^{2} / 3$ [11], an increase in the amplitude of the oscillations $r_{\beta}$ will lead to a considerable increase in the radiated $\mathrm{x}$-ray power. We can attribute this increase in power to the hosing instability rather than resonant interaction with the laser fields $[15,25]$ because it occurs at a long propagation distance past a few dephasing lengths.

Reference [19] describes how coupled motion between an electron beam that is off center in a preformed ion channel and the surrounding plasma sheath electrons may lead to an amplification of an initial transverse offset $x_{0}$ by a factor $x(z, \xi) / x_{0}(\xi) \simeq 0.34 A^{-3 / 2} e^{A} \cos \left[k_{\beta} z-A / \sqrt{3}+\pi / 4\right]$, where the growth factor, $A(z, \xi)$, depends on both the axial displacement $z$, and the "beam coordinate" $\xi=t-z / c$, as $[19,21,32] A(z, \xi)=1.3\left[c_{r} c_{\psi}\left(k_{\beta} z\right)^{2}\left(\omega_{0} \xi\right)\right]^{1 / 3}$, where $k_{\beta}=$ $k_{p} / \sqrt{2 \gamma}\left(k_{p}\right.$ is plasma wave number and $\gamma$ is the Lorentz factor), $\omega_{0}=k_{p} / \sqrt{2}$. $c_{r}$ is a reduction factor for the case of

\footnotetext{
${ }^{1}$ The dephasing length is the distance for the electron beam to advance through half the accelerating structure.
}

nonadiabatic ion-channel formation, varying along the beam and $c_{\psi}$ is a reduction factor for the case when the plasma electron velocity is large and its magnetic field becomes significant [21].

The growth length $L_{g}=z / A^{3 / 2} \approx 0.1 \lambda_{\beta} / \sqrt{c_{r} c_{\psi} \omega_{0} \xi}$. For our parameters, $L_{g} \approx 0.3 \lambda_{\beta}$ for the rear of the beam and $\lambda_{\beta} \approx 300 \mu \mathrm{m}$, meaning the expected growth length for the instability is only $\sim 100 \mu \mathrm{m}$ and therefore an order of magnitude growth of the offset is expected after a $\mathrm{mm}$ propagation. The instability will saturate once the hosing amplitude reaches of order the channel radius. This is broadly consistent with the experimental measurements if the increase in $\mathrm{x}$-ray flux is due to hosing commencing after twice the dephasing limit assuming that this is measured from the end of the injection stage. Recent theoretical studies have shown that the hosing instability may be damped by phase mixing for broad energy spread beams [33], however, if the growth rate is very large relative to the betatron frequency and the seed amplitude is large, the hosing instability is still significant.

OsIRIS particle-in-cell (PIC) simulations were conducted to examine the electron acceleration process and to better understand the $\mathrm{x}$-ray radiation enhancement. The simulations were conducted with a preionized gas ( $\mathrm{n}=0.005 \mathrm{n}_{\text {crit }}$, where $\mathrm{n}_{\mathrm{crit}}=\epsilon m_{e} \omega_{0}^{2} / e^{2}$ is the critical plasma density). The simulation $\left[(82.8 \times 82.8 \times 82.8) \mu \mathrm{m}^{3}\right]$ box was set up in the comoving frame of the laser, which propagates in the $+z$-direction and is polarized in the $y$-direction. The longitudinal and transverse grid resolution is 31 and 4 cells per laser wavelength, respectively. Absorbing boundary conditions were utilized to prevent electrons reflecting at boundaries. The simulated laser pulse had a 28 fs FWHM pulse duration and focused intensity of $3.4 \times 10^{19} \mathrm{~W} \mathrm{~cm}^{-2}$ with waist size $w_{0} \approx 10 \mu \mathrm{m}$.

In order to perform in situ radiation simulations, we implemented a Monte Carlo algorithm based method for calculating the classical synchrotron spectrum of the charged particles. This algorithm works by calculating the standard textbook two-dimensional probability distribution for charged particles in instantaneously relativistic motion [34] to generate macrophotons with randomly determined normalized frequency/normalized angle pair $(\tilde{\omega}, \tilde{\theta})$ for each radiating particle at a particular time step. Whether a particle radiates or not is determined by a minimum Lorentz factor and minimum critical frequency $\omega_{\text {crit }}$ based on their trajectory over a time step. We set $\hbar \omega_{\text {crit }}=1.56 \mathrm{keV}$ as the critical frequency of allowed photons $\left(\omega_{\text {crit }}=3 \gamma^{3} c / \varrho\right.$, where $\varrho$ is the radius of curvature). The emitted macrophotons have the energy that the particle would have radiated over the time step. In the limit of sufficiently large numbers of photons, the algorithm recovers the correct synchrotron spectrum.

Figures 3(a)-3(e) show the time-dependent evolution of electron energy, electron radius of curvature $\left(r_{c v e}\right)$, photon frequency (energy), photon emission angle $\theta_{x}$ integrated 


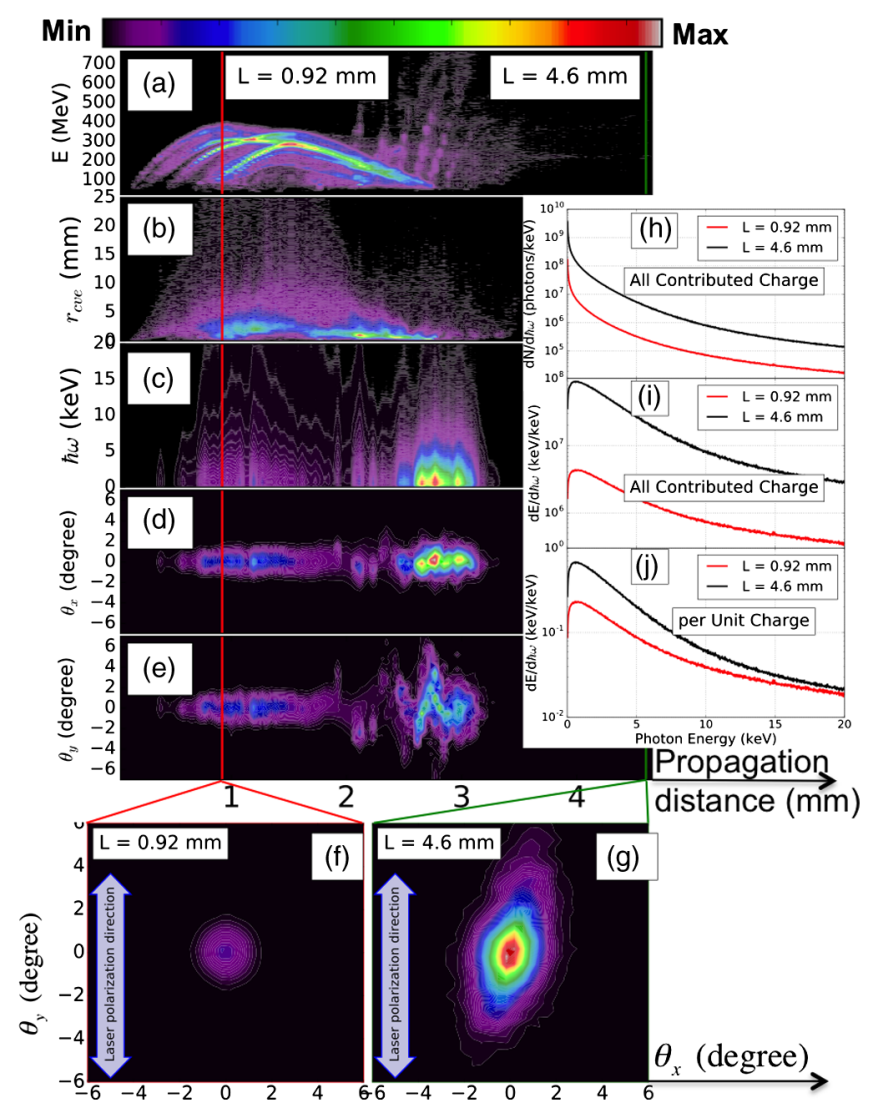

FIG. 3. Contour plots of phase space density as a function of propagation distance $z$ of (a) electron energy (only those that contribute to the radiation emission), (b) electron radius of curvature $\left(r_{c v e}\right)$ at point of emission, (c) photon energy $(\mathrm{keV})$, (d) photon emission angle $\theta_{x}$ integrated over $\theta_{y}$, and (e) photon emission angle $\theta_{y}$ integrated over $\theta_{x}$. Parts (f) and (g) show the time integrated x-ray angular distribution at 0.92 and $4.6 \mathrm{~mm}$, respectively. The time integrated $\mathrm{x}$-ray spectra of all contributed charge [(h) dN/d $\hbar \omega$ and (i) $\mathrm{dE} / \mathrm{d} \hbar \omega$ vs $\hbar \omega$, respectively] and per unit charge $[(\mathrm{j}) \mathrm{dE} / \mathrm{d} \hbar \omega$ vs $\hbar \omega]$ at 0.92 and $4.6 \mathrm{~mm}$, respectively.

over $\theta_{y}$, and photon emission angle $\theta_{y}$ integrated over $\theta_{x}$. In Fig. 3(a), a large group of electrons is initially injected and accelerated in the LWFA regime to energies of several hundred $\mathrm{MeV}$. After propagating past the dephasing length $(1.3 \mathrm{~mm})$, the electrons start to decelerate. When the electron beam catches up with the laser pulse at $L=2.8 \mathrm{~mm}$, the net energy gain of the electrons causes pump depletion and the ponderomotive force of the laser is unable to sustain the wakefield. The wakefield becomes beam driven and is capable of trapping and accelerating a secondary bunch of electrons to almost $\mathrm{GeV}$ energies. The relatively low density of high energy electrons can be partially caused by the absorbing boundary conditions, which allow electrons to escape.

In Fig. 3(b), the electron radius of curvature is observed to decrease with propagation distance. Initially, the radius of curvature is large and the electrons oscillate with a small amplitude in the undulator regime. As the electrons interact with the laser pulse and the electron hosing instability sets in, the electron oscillations increase with a corresponding decrease in the radius of curvature, signifying radiation emission in the wiggler regime [3].

In Figs. 3(c)-3(e), the photon energy and the integrated angular distributions are plotted as a function of the propagation distance. The photon energy initially increases and decreases in lockstep with the acceleration and deceleration of the electron beam in the LWFA regime. Afterwards, a significant enhancement occurs for the photon energy due to the acceleration of the secondary electron bunch and its interaction with the laser pulse, which provides a seed perturbation for the electron hosing instability [35]. Figures 3(a) and 3(c) are consistent with the experimental data shown in Fig. 2. Figures 3(d) and 3(e) show that the angular distributions of the photons are relatively isotropic in the earlier stages of the wakefield evolution and signify electrons oscillating in the undulator regime. As the simulation progresses, the distribution becomes anisotropic, especially in the $\theta_{y}$ (i.e., laser polarization) direction. The angular deflection in $\theta_{y}$ reaches approximately $\pm 6^{\circ}$, which is almost 3 times larger than the deflection in $\theta_{x}$. The increased angular deflection in the $\theta_{y}$ direction indicates the onset of the electron hosing instability. The corresponding increase in the photon energy and decrease in the electron radius of curvature can be seen in Figs. 3(c) and 3(b), respectively.

The integrated angular distributions of the radiated photons are shown in Figs. 3(f) and 3(g). The angular profile of the photons evolves from an isotropic distribution (bottom left) to one that is anisotropic in the laser polarization direction (bottom right). Both the anisotropy and high intensity of the photon angular distribution are caused by the electron hosing instability, which causes the electron beam to emit more radiation at each point in its oscillating trajectory. The integrated $\mathrm{x}$-ray spectra are shown in Figs. 3(h)-3(j), and mirror the significant increase in both the number of photons and the radiated photon energy (per unit charge) after the instability sets in, consistent with the trend shown in Fig. 2(b).

A 3D charge-density map showing the evolution of the wakefield at different lengths is given in Fig. 4. In Fig. 4(a), the wakefield is in the LWFA regime and electrons are continuously injected into the first bubble. A clear electron beam is formed as seen in the projected images. As this first group of electrons catches up and interacts with the laser pulse front, Fig. 4(b), the electrons undergo increased oscillations in the laser polarization direction. This motion disrupts the spherical shape of the cavity, especially towards the cavity rear. In the $y-z$ plane, a noticeable deviation of the electron beam from the cavity axis can be seen. This slight deviation plants the seed for the electron hosing instability [35]. A secondary group of electrons also forms at the rear of the cavity in Fig. 4(b), which is then accelerated by the primary bunch after a transition from an LWFA to a PWFA occurs [18]. 


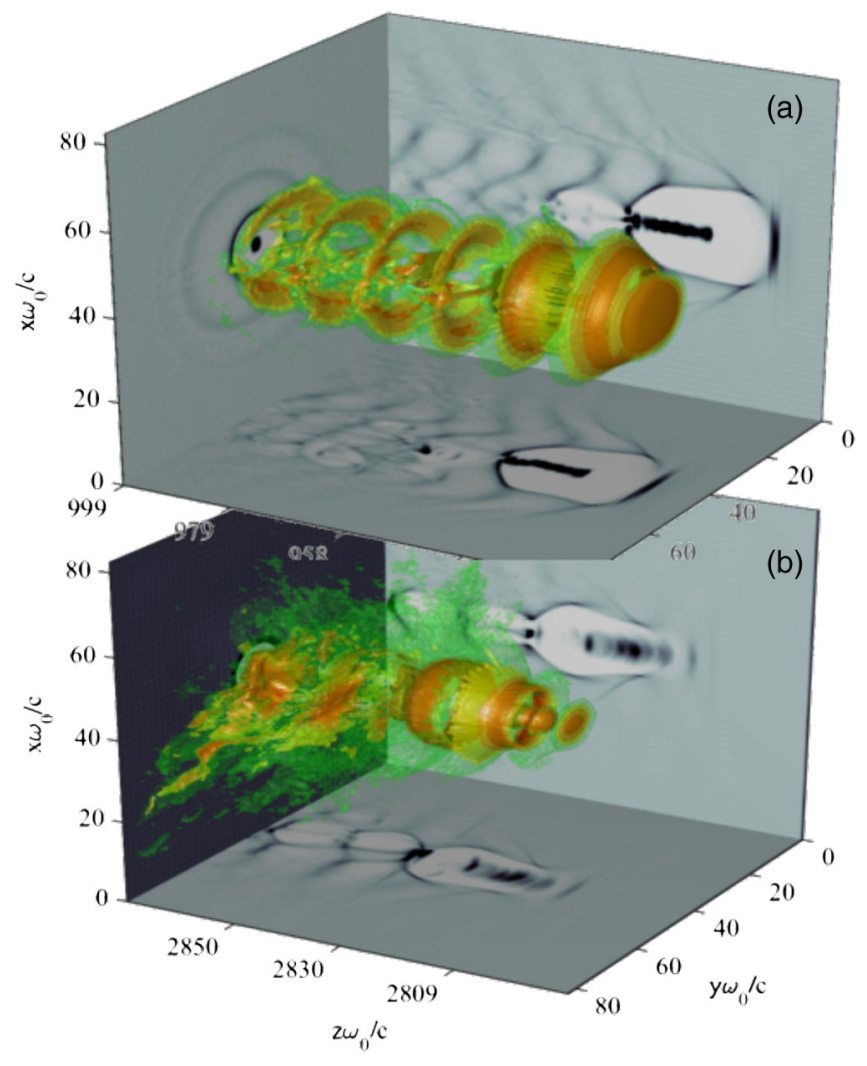

FIG. 4. 3D map of charge density and its projection on the $x-z$ and $y-z$ planes. At (a) $z=0.92 \mathrm{~mm}$, (b) $z=2.8 \mathrm{~mm}$.

In conclusion, an enhancement in the x-ray emission resulting from the electron hosing instability has been demonstrated. Both experimental and 3D PIC simulation results show that the total radiated photon energy starts to increase shortly after the electron beam catches up with the laser pulse. The interaction of the primary electron beam with the laser pulse provides a seed perturbation for the electron hosing instability and thus significantly enhances the x-ray radiation by almost an order of magnitude. Subsequently, the wakefield driven by the high-energy electron beam can trap and accelerate a secondary group of electrons, during which a transition from an LWFA to a PWFA occurs. Furthermore, this enhancement cannot be attributed to simply an increase in the total number of accelerated charges with length. The onset of the instability can also be inferred from the simulations by observing the evolution of the emitted photon angular distribution and the electron radius of curvature. The photon angular distribution changes from an approximately isotropic distribution to an anisotropic distribution, where the emitted angle $\theta_{y}$ (in the laser polarization direction) is about 3 times larger than $\theta_{x}$, with a corresponding increase in the x-ray source size. At the same time, the electron radius of curvature shows a negative linear correlation with the variation of $\theta_{y}$ due to the transition from the undulator regime to the wiggler regime. The greatly enhanced $\mathrm{x}$-ray radiation can be achieved by taking advantage of a naturally occurring instability in wakefield acceleration and is useful for applications where a high x-ray flux is required with minimal change to experimental design.

\section{ACKNOWLEDGMENTS}

This work is supported by the U.S. Department of Energy/National Nuclear Security Administration Grant No. DE-NA0002372, the National Science Foundation Career Grant No. 1054164, and the Air Force Office of Scientific Research Young Investigator Program Grant No. FA9550-12-1-0310. The author would like to acknowledge the OSIRIS consortium for use of the OSIRIS 2.0 framework.

[1] X. Wang et al., Quasi-monoenergetic laser-plasma acceleration of electrons to $2 \mathrm{GeV}$, Nat. Commun. 4, 1988 (2013).

[2] W. P. Leemans, A. J. Gonsalves, H. S. Mao et al., Multi$\mathrm{GeV}$ Electron Beams from Capillary-Discharge-Guided Subpetawatt Laser Pulses in the Self-Trapping Regime, Phys. Rev. Lett. 113, 245002 (2014).

[3] S. Corde, K. Ta Phuoc, G. Lambert, R. Fitour, V. Malka, A. Rousse, A. Beck, and E. Lefebvre, Femtosecond x rays from laser-plasma accelerators, Rev. Mod. Phys. 85, 1 (2013).

[4] S. Corde, C. Thaury, K. Ta Phuoc et al., Betatron emission as a diagnostic for injection and acceleration mechanisms in laser plasma accelerators, Plasma Phys. Controlled Fusion 54, 124023 (2012).

[5] S. Kneip, C. McGuffey, J. L. Martins et al., Characterization of transverse beam emittance of electrons from a laserplasma wakefield accelerator in the bubble regime using betatron x-ray radiation, Phys. Rev. ST Accel. Beams 15, 021302 (2012).

[6] M. Schnell, A. Savert, B. Landgraf et al., Deducing the Electron-Beam Diameter in a Laser-Plasma Accelerator Using X-Ray Betatron Radiation, Phys. Rev. Lett. 108, 075001 (2012).

[7] T.Z. Zhao, K. Behm, C. F. Dong, X. Davoine, S. Y. Kalmykov, V. Petrov, V. Chvykov, P. Cummings, B. Hou, A. Maksimchuk, J. A. Nees, V. Yanovsky, A. G. R. Thomas, and K. Krushelnick, High-Flux Femtosecond X-Ray Emission from Controlled Generation of Annular Electron Beams in a Laser Wakefield Accelerator, Phys. Rev. Lett. 117, 094801 (2016).

[8] A. Rousse, K. T. Phuoc, R. Shah et al., Production of a keV $\mathrm{X}$-Ray Beam from Synchrotron Radiation in Relativistic Laser-Plasma Interaction, Phys. Rev. Lett. 93, 135005 (2004).

[9] K. T. Phuoc, F. Burgy, J.-P. Rousseau, V. Malka, A. Rousse, R. Shah, D. Umstadter, A. Pukhov, and S. Kiselev, Laser based synchrotron radiation, Phys. Plasmas 12, 023101 (2005).

[10] S. Kneip, C. McGuffey, J. L. Martins, S. F. Martins, C. Bellei, V. Chvykov, F. Dollar, R. Fonseca, C. Huntington, 
G. Kalintchenko et al., Bright spatially coherent synchrotron X-rays from a table-top source, Nat. Phys. 6, 980 (2010).

[11] E. Esarey, B. A. Shadwick, P. Catravas, and W. P. Leemans, Synchrotron radiation from electron beams in plasmafocusing channels, Phys. Rev. E 65, 056505 (2002).

[12] I. Kostyukov, S. Kiselev, and A. Pukhov, X-ray generation in an ion channel, Phys. Plasmas 10, 4818 (2003).

[13] K. T. Phuoc, E. Esarey, V. Leurent, E. Cormier-Michel, C. G. R. Geddes, C. B. Schroeder, A. Rousse, and W. P. Leemans, Betatron radiation from density tailored plasmas, Phys. Plasmas 15, 063102 (2008).

[14] K. T. Phuoc, S. Corde, R. Fitour, R. Shah, F. Albert, J.-P. Rousseau, F. Burgy, A. Rousse, V. Seredov, and A. Pukhov, Analysis of wakefield electron orbits in plasma wiggler, Phys. Plasmas 15, 073106 (2008).

[15] A. G. R. Thomas and K. Krushelnick, Betatron X-ray generation from electrons accelerated in a plasma cavity in the presence of laser fields, Phys. Plasmas 16, 103103 (2009).

[16] A. G. R. Thomas, Scalings for radiation from plasma bubbles, Phys. Plasmas 17, 056708 (2010).

[17] O. Lundh, J. Lim, C. Rechatin, L. Ammoura, A. Ben-Ismal, X. Davoine, G. Gallot, J-P. Goddet, E. Lefebvre, V. Malka, and J. Faure, Few femtosecond, few kiloampere electron bunch produced by a laser-plasma accelerator, Nat. Phys. 7, 219 (2011).

[18] P. E. Masson-Laborde, M.Z. Mo, A. Ali et al., Gigaelectronvolt electrons due to a transition from laser wakefield acceleration to plasma wakefield acceleration, Phys. Plasmas 21, 123113 (2014).

[19] D. H. Whittum, W. M. Sharp, S. S. Yu, M. Lampe, and G. Joyce, Electron-Hose Instability in the Ion-Focused Regime, Phys. Rev. Lett. 67, 991 (1991).

[20] E. S. Dodd, R. G. Hemker, C.-K. Huang, S. Wang, C. Ren, W. B. Mori, S. Lee, and T. Katsouleas, Hosing and Sloshing of Short-Pulse GeV-Class Wakefield Drivers, Phys. Rev. Lett. 88, 125001 (2002).

[21] C. Huang, W. Lu, M. Zhou et al., Hosing Instability in the Blow-Out Regime for Plasma-Wakefield Acceleration, Phys. Rev. Lett. 99, 255001 (2007).

[22] C. M. Huntington, A. G. R. Thomas, C. McGuffey et al., Current Filamentation Instability in Laser Wakefield Accelerators, Phys. Rev. Lett. 106, 105001 (2011).

[23] M. C. Kaluza, S. P. D. Mangles, A. G. R. Thomas et al., Observation of a Long-Wavelength Hosing Modulation of a High-Intensity Laser Pulse in Underdense Plasma, Phys. Rev. Lett. 105, 095003 (2010).

[24] Y. Ma et al., Generation of femtosecond $\gamma$-ray bursts stimulated by laser-driven hosing evolution, Sci. Rep. 6, 30491 (2016).

[25] S. Cipiccia, M. R. Islam, B. Ersfeld et al., Gamma-rays from harmonically resonant betatron oscillations in a plasma wake, Nat. Phys. 7, 867 (2011).

[26] Y. Y. Lau, Classification of Beam Breakup Instabilities in Linear Accelerators, Phys. Rev. Lett. 63, 1141 (1989).

[27] M. Vargas et al., Improvements to laser wakefield accelerated electron beam stability, divergence, and energy spread using three-dimensional printed two-stage gas cell targets, Appl. Phys. Lett. 104, 174103 (2014).

[28] C. McGuffey, A. G. R. Thomas, W. Schumaker et al., Ionization Induced Trapping in a Laser Wakefield Accelerator, Phys. Rev. Lett. 104, 025004 (2010).

[29] S. Fourmaux, S. Corde, K. Ta Phuoc et al., Demonstration of the synchrotron-type spectrum of laser-produced Betatron radiation, New J. Phys. 13, 033017 (2011).

[30] K. T. Behm, T.Z. Zhao, J. M. Cole, A. Maksimchuk, S. P. D. Mangles, J. Nees, J. C. Wood, V. Yanovsky, K. Krushelnick, and A. G. R. Thomas, Ionization injection effects in X-ray spectra generated by betatron oscillations in a laser wakefield accelerator, Plasma Phys. Controlled Fusion 58, 055012 (2016).

[31] W. Lu, M. Tzoufras, C. Joshi, F. S. Tsung, W. B. Mori, J. Vieira, R. A. Fonseca, and L. O. Silva, Generating multi$\mathrm{GeV}$ electron bunches using single stage laser wakefield acceleration in a 3D nonlinear regime, Phys. Rev. ST Accel. Beams 10, 061301 (2007).

[32] A. A. Geraci and D. H. Whittum, Transverse dynamics of a relativistic electron beam in an underdense plasma channel, Phys. Plasmas 7, 3431 (2000).

[33] T. J. Mehrling, R. A. Fonseca, A. Martinez de la Ossa, and J. Vieira, Mitigation of the Hose Instability in PlasmaWakefield Accelerators, Phys. Rev. Lett. 118, 174801 (2017).

[34] J. D. Jackson, Classical Electrodynamics (Wiley, New York, 1975).

[35] See Supplemental Material http://link.aps.org/ supplemental/10.1103/PhysRevAccelBeams.21.041303 for the evolution of the electron beam hosing instability over long propagation distances of from OSIRIS particle-incell simulations. 